\title{
Analysis and Design of Vertical-Cavity Surface-Emitting Lasers for Self-Sustained Pulsation Operation
}

\author{
S. F. Yu
}

\begin{abstract}
The generation mechanism of self-sustained pulsation in vertical-cavity surface-emitting lasers (VCSEL's) is analyzed with the influence of lateral loss effects, such as selffocusing, lateral diffraction loss, and spatial hole burning, taken into consideration. The condition for self-sustained pulsation is also derived in an analytical form. It is shown that the lateral loss effects have significant influence on the excitation of self-sustained pulsation, especially for devices with small size. Furthermore, the possibility of using diffused quantum-well (QW) structures in VCSEL's to enhance self-sustained pulsation is studied. It is found that by using diffused QW structures, the range of core area to obtain self-sustained pulsation is increased. In addition, peak power and repetition frequency of the pulses can also be improved.
\end{abstract}

Index Terms - Laser stability, semiconductor device modeling, semiconductor lasers, surface-emitting lasers.

\section{INTRODUCTION}

C IRCULAR output beam with low divergence, high modulation speed, low threshold current, and ease of fabrication in two-dimensional (2-D) array are the attractive features of vertical-cavity surface-emitting lasers (VCSEL's) for the application in optical interconnection, switching, and compact disk systems. In order to utilize VCSEL's in these optical data storage and processing systems, it is required to reduce the influence of optical feedback noise [1]. One of the possible methods to reduce feedback noise is to employ the phenomenon of self-sustained pulsation (SSP) in semiconductor lasers [2], [3].

It is known that edge-emitting lasers with narrow-stripe geometry exhibit SSP due to the saturable absorption arising from an unpumped active layer along the longitudinal direction [4]. Therefore, VCSEL's with similar lateral confinement structures, such as those with selective oxidization [5], proton[6], [7] or $\mathrm{H}^{+}$-implanted [8] configurations, are expected to support SSP. However, to the best of our knowledge, similar SSP operation in VCSEL's has not been observed experimentally. The reason for the suppression of SSP operation may be attributed to the small cavity size of VCSEL's such that the lateral loss effects (i.e., self-focusing, diffraction loss, spatial hole burning, and thermal lensing) dominate over the lateral overlap effects (i.e., overlap volume between the saturable region and the lasing mode) [2], [3]. Therefore, the

Manuscript received March 3, 1997; revised November 12, 1997. This work was supported by the HKU CRCG.

The author is with the Department of Electrical and Electronic Engineering, University of Hong Kong, Pokfulam Road, Hong Kong.

Publisher Item Identifier S 0018-9197(98)01767-9. requirement of high saturable absorption is not sufficient to excite SSP operation in VCSEL's and the influence of lateral loss has to be taken into consideration.

Recent development of index or gain-guiding VCSEL's [5]-[8] mainly emphasize low threshold, narrow output beam, and single-mode operation. However, the structures of these devices may not be suitable for supporting SSP operation due to the lack of control of lateral loss and lateral overlap. In order to minimize lateral loss effects and to maintain sufficient saturable absorption, a novel structure of VCSEL's is required. Therefore, we suggest the use of selectively impurity-induced compositional disordering in quantum-well $(\mathrm{QW})$ active layer [9]: 1) to reduce the influence of lateral loss effects and 2) to control the overlap volume between the absorption regions and the lasing mode in VCSEL's.

In this paper, the influence of lateral loss effects on the generation mechanism of SSP in VCSEL's is studied theoretically. In addition, the possibility of using diffused quantum-well (DFQW) structures to enhance SSP operation in VCSEL's is also investigated. In Section II, the condition for SSP is derived with self-focusing and lateral diffraction loss taken into consideration. Furthermore, a numerical model of VCSEL's is developed to include the influence of spatial hole burning. In Section III, VCSEL with DFQW structure is proposed. The influence of impurity-induced compositional disordering on the optical gain and refractive index of QW materials is given. Also, the generation mechanism of SSP using DFQW structures is explained. In Section IV, the conditions for achieving SSP in DFQW VCSEL's are investigated. A brief discussion and conclusion are given in Section V.

\section{Theoretical Analysis of Self-Sustained Pulsation}

\section{A. Condition of Self-Sustained Pulsation in VCSEL's}

The condition of SSP in VCSEL's including self-focusing and diffraction loss can be derived from the modified rate equations given below:

$$
\begin{aligned}
\frac{\partial S(t)}{\partial t}= & \nu_{g}\left(\Gamma_{1}\left(N_{1,2}\right) G_{1}\left(N_{1}\right)+\Gamma_{2}\left(N_{1,2}\right) G_{2}\left(N_{2}\right)\right. \\
& -\alpha\left(N_{1,2}\right) S(t)+R_{\mathrm{sp}}\left(N_{1,2}\right) \\
\frac{\partial N_{1}(t)}{\partial t}= & \frac{J(t)}{q d}-\frac{N_{1}(t)}{\tau_{1}}-\nu_{g} \Gamma_{1}\left(N_{1,2}\right) G_{1}\left(N_{1}\right) S(t) \\
\frac{\partial N_{2}(t)}{\partial t}= & -\frac{N_{2}(t)}{\tau_{2}}-\nu_{g} \Gamma_{2}\left(N_{1,2}\right) G_{2}\left(N_{2}\right) S(t)
\end{aligned}
$$


where $S$ is the effective photon density, $N_{1}$ and $N_{2}$ are the equivalent carrier concentration inside the core (gain) and cladding (absorption) region of the laser, respectively, $\tau_{1}$ and $\tau_{2}$ are the corresponding carrier lifetime, $\nu_{g}$ is the group velocity, $R_{\mathrm{sp}}$ is the spontaneous emission term, $J$ is the injection current density, $q$ is the electron charge, and $d$ is the thickness of the active layer.

The influence of self-focusing is introduced into the model through the carrier-dependent confinement factors, $\Gamma_{1}$ and $\Gamma_{2}$ [10]. This is because the lateral field profile (i.e., lateral confinement factor) is varied due to carrier-induced index change inside the active layer. The influence of diffraction loss is also included into the model through the carrier dependent cavity loss, $\alpha$. This is because the reflectivity of Bragg reflectors (which is inversely proportional to the cavity loss) varies with the penetration depth (i.e., effective guide width) of the lateral field [11].

For laser in SSP operation, the carrier concentration inside the gain $\left(N_{1}\right)$ region and absorption region $\left(N_{2}\right)$ vary out of phase [12]. This is because as $N_{1}$ approaches its threshold, photon density starts to generate and is absorbed in the absorption region. Hence, $N_{2}$ in the absorption region is increased due to the absorption. However, $N_{1}$ is reduced with the stimulated emission. Therefore, it is possible to simplify our analysis by relating the net change of carrier concentration inside the active $\left(\Delta N_{1}\right)$ and absorption $\left(\Delta N_{2}\right)$ regions to the net change of equivalent carrier concentration, $\Delta N$, as below

$$
\Delta N \equiv \Delta N_{1} \approx-\Delta N_{2} \text {. }
$$

The change of optical gain, $\Delta G_{1}$ and $\Delta G_{2}$, in the active and absorption regions, respectively, can be expressed as

$$
\Delta G_{1}=\frac{\partial G_{1}}{\partial N_{1}} \Delta N_{1} \equiv \frac{\partial G_{1}}{\partial N} \Delta N
$$

and

$$
\Delta G_{2}=\frac{\partial G_{2}}{\partial N_{2}} \Delta N_{2} \equiv-\frac{\partial G_{2}}{\partial N} \Delta N
$$

where $\partial G_{1} / \partial N$ and $\partial G_{2} / \partial N$ are the corresponding differential gain. The carrier-induced change in confinement factors are given by

and

$$
\Delta \Gamma_{1}=\frac{\partial \Gamma_{1}}{\partial N_{1}} \Delta N_{1}+\frac{\partial \Gamma_{1}}{\partial N_{2}} \Delta N_{2} \equiv \frac{\partial \Gamma}{\partial N} \Delta N
$$

$$
\Delta \Gamma_{2}=\frac{\partial \Gamma_{2}}{\partial N_{1}} \Delta N_{1}+\frac{\partial \Gamma_{2}}{\partial N_{2}} \Delta N_{2} \equiv-\frac{\partial \Gamma}{\partial N} \Delta N
$$

where we have assumed $\Gamma_{1}+\Gamma_{2}=1$ and $\partial \Gamma / \partial N$ is defined as the equivalent differential confinement factor. The change of cavity loss can be written as

$$
\Delta \alpha=\frac{\partial \alpha}{\partial N_{1}} \Delta N_{1}+\frac{\partial \alpha}{\partial N_{2}} \Delta N_{2} \equiv \frac{\partial \alpha}{\partial N} \Delta N
$$

where $\partial \alpha / \partial N$ is defined as the equivalent differential cavity loss. Substituting (4)-(7) into (1)-(3) gives [10]

$$
\begin{aligned}
\frac{\partial \Delta S}{\partial t}= & \nu_{g} S_{0}\left(\left(G_{10}-G_{20}\right) \frac{\partial \Gamma}{\partial N}+\left(\Gamma_{10} \frac{\partial G_{1}}{\partial N}-\Gamma_{20} \frac{\partial G_{2}}{\partial N}\right)\right. \\
& \left.\quad-\frac{\partial \alpha}{\partial N}\right) \Delta N \\
\frac{\partial \Delta N}{\partial t}= & -\left(\frac{1}{\tau_{1}}+\nu_{g} S_{0}\left\{G_{10} \frac{\partial \Gamma}{\partial N}+\Gamma_{10} \frac{\partial G_{1}}{\partial N}\right\}\right) \Delta N \\
& -\nu_{g} \Gamma_{10} G_{10} \Delta S \\
-\frac{\partial \Delta N}{\partial t}= & \left(\frac{1}{\tau_{2}}+\nu_{g} S_{0}\left\{G_{20} \frac{\partial \Gamma}{\partial N}+\Gamma_{20} \frac{\partial G_{2}}{\partial N}\right\}\right) \Delta N \\
& -\nu_{g} \Gamma_{20} G_{20} \Delta S
\end{aligned}
$$

where the subscript 0 stands for steady state and $\Delta S$ is the small change of photon density. In the derivation of (8)-(10), the spontaneous emission term, $R_{\mathrm{sp}}$, is ignored in the calculation.

$\Delta N$ can be eliminated from (8) by adding (9) and (10) and after substituting the expression of $\Delta N$ back into (8), we get (11), shown at the bottom of the page, where $\alpha_{0}$ is the cavity loss at steady state. For unstable operation (i.e., SSP), $\Delta S$ should increase with time and the factor in front of $\Delta S$ must be positive.

The carrier lifetime inside the active region should be much shorter than that inside the absorption region due to the high injection of carrier concentration such that $1 / \tau_{1}-1 / \tau_{2}>0$. For deviced with weak lateral confinement (i.e., $\Gamma_{2}>\Gamma_{1}$ ), $\Gamma_{10} \partial G_{1} / \partial N-\Gamma_{20} \partial G_{2} / \partial N<0$ is satisfied as the differential gain of the absorption region is relatively higher than that of the gain region. However, the denominator of the factor can be assumed to be greater than zero. This is because SSP only occurs for the device biased near threshold and this implies that the steady-state value of $S_{0}$ is very small. Therefore, the condition of SSP is reduced to

$$
\eta \equiv\left(G_{10}-G_{20}\right) \underbrace{\frac{\partial \Gamma}{\partial N}}_{(1)}+\underbrace{\left(\Gamma_{10} \frac{\partial G_{1}}{\partial N}-\Gamma_{20} \frac{\partial G_{2}}{\partial N}\right)}_{(2)}-\underbrace{\frac{\partial \alpha}{\partial N}}_{(3)}<0
$$

where $\eta$ is the instability constant. In (12), $\left(G_{10}-G_{20}\right)>0$ because $G_{20}$ is the optical loss in the absorption region. Now, the condition of SSP is determined by the sign of: 1) the differential confinement factor arising from the self-focusing effect; 2) the differential gain difference between the gain and absorption regions; and 3 ) the differential cavity loss arising from the diffraction loss.

The guiding structure of lasers determines the sign of $\partial \Gamma / \partial N$. It is shown in [10] that $\partial \Gamma / \partial N$ has a negative

$$
\frac{\partial \Delta S}{\partial t}=-\nu_{g} \alpha_{0} \frac{\nu_{g} S_{0}\left(\left(G_{10}-G_{20}\right) \frac{\partial \Gamma}{\partial N}+\left(\Gamma_{10} \frac{\partial G_{1}}{\partial N}-\Gamma_{20} \frac{\partial G_{2}}{\partial N}\right)-\frac{\partial \alpha}{\partial N}\right)}{\frac{1}{\tau_{1}}-\frac{1}{\tau_{2}}+\nu_{g} S_{0}\left(\left(G_{10}-G_{20}\right) \frac{\partial \Gamma}{\partial N}+\left(\Gamma_{10} \frac{\partial G_{1}}{\partial N}-\Gamma_{20} \frac{\partial G_{2}}{\partial N}\right)\right)} \Delta S
$$


value for index guiding but a positive value for gain or antiindex guiding devices such that the device with index-guiding structure enhances SSP. In fact, it is shown in [13] that an edge-emitting device with a strong index-guiding structure enhances SSP through the presence of self-focusing effect (i.e., $\partial \Gamma / \partial N)$. In [13, Fig. 2], it is observed that the reduction of mode gain (i.e., reduction of carrier concentration) decreases the FWHM of the optical field (i.e., increases the lateral confinement of the optical mode) which implies that $\partial \Gamma / \partial N$ has a negative value. Therefore, the numerical analysis in [13] verifies (12) in explaining the generation mechanism of SSP.

It seems that oxide-confined VCSEL's have much stronger index-guiding structures than those in edge-emitting lasers and SSP operation can be easily achieved by the negative value of $\partial \Gamma / \partial N$. However, SSP similar to that occurred in edge-emitting lasers [13] has not been observed in VCSEL's and the reason can be explained by the presence of $\partial \alpha / \partial N$ in (12). It can be shown that the diffraction loss increases with the reduction of the effective lateral guide-width (i.e., the effective aperture of the core region) of the devices [14]. This implies that the cavity loss, $\alpha$, is proportional to the refractive index inside the core region (or inversely proportional to the refractive index inside the cladding region) and $\partial \alpha / \partial N$ has a negative value. Therefore, the diffraction loss works against the generation of SSP in VCSEL's as indicated in (12).

In the derivation of (12), we have assumed that the small variation of $\Delta N_{1}$ and $\Delta N_{2}$ are out of phase but equal in magnitude. However, the magnitude of $\Delta N_{1}$ and $\Delta N_{2}$ may be different due to the mixing effect arising from the diffusion of carriers between the active and absorption regions [12]. The influence of the mixing effect on (12) can be checked by comparing with the SSP condition of edge-emitting lasers. If the lateral loss effects are ignored in the derivation of (12), the condition of SSP reduces to $\eta_{a}=\Gamma_{10} \partial G_{1} / \partial N$ $\Gamma_{20} \partial G_{2} / \partial N$. The condition of SSP given in [15] is equal to $\eta_{b}=\Gamma_{10} G_{10} \partial G_{1} / \partial N-\Gamma_{20}\left|G_{20}\right| \partial G_{2} / \partial N$ where two extra terms, $G_{10}$ and $G_{20}$, are observed in the expression of $\eta_{b}$. In order to take into account the influence of the mixing effect, the condition of SSP should be modified as below:

$$
\begin{aligned}
\eta= & \left(G_{10}-G_{20}\right) \frac{\partial \Gamma}{\partial N} \\
& +\left(\Gamma_{10} \frac{\partial G_{1}}{\partial N}-\gamma_{0} \Gamma_{20} \frac{\partial G_{2}}{\partial N}\right)-\frac{\partial \alpha}{\partial N}<0
\end{aligned}
$$

where $\gamma_{0}=\left|G_{20} / G_{10}\right|$.

\section{B. Numerical Model of VCSEL's Including Lateral Loss}

In the above analytical analysis of SSP, the influence of thermal lensing and lateral spatial hole burning has been ignored in order to simplify our derivation. In the following development of the numerical model of VCSEL's, the influence of lateral spatial hole burning (as well as self-focusing and diffraction loss) will be taken into consideration. However, the thermal lensing effect is ignored in our analysis because: 1) the response time of heat flow is much longer than the time variation of carrier concentration and photon density and 2) the thermal lensing effect is less dominant for the device biased close to its threshold.
The modified rate-equation model of VCSEL's is given by [16]

$$
\begin{aligned}
\frac{\partial N(r, t)}{\partial t}= & \frac{J(r, t)}{q d}-\frac{N(r, t)}{\tau}-\nu_{g} \Gamma_{z} g(N)|\Psi(r, t)|^{2} S(t) \\
& +D \frac{1}{r} \frac{\partial}{\partial r}\left(r \frac{\partial N(r, t)}{\partial r}\right) \\
\frac{\partial S(t)}{\partial t}= & \nu_{g}\left(\Gamma_{z} G-\alpha_{m}-\alpha(t)\right) S(t)+R_{\mathrm{sp}}(t)
\end{aligned}
$$

where $N$ is the lateral distribution of carrier concentration inside the active layer, $\Gamma_{z}$ is the longitudinal confinement factor, $D$ is the ambipolar diffusion coefficient, and $g$ is the optical gain of the QW active layer to be determined. The symbol $\Psi$ represents the normalized lateral field (i.e., $1=2 \int_{0}^{\infty}|\Psi|^{2} r d r / W^{2}$ ) of the fundamental mode (i.e., $\mathrm{LP}_{01}$ mode) with $S$ as the corresponding photon density and $W$ as the radius of the core region. In (14) and (15), the self-focusing effect is directly introduced into the computation through the time dependence of $\Psi$. The time variation of $\Psi$ can be calculated by solving the complex scalar Helmholtz equation (see Appendix A) with the influence of nonuniformly lateral distribution of refractive index taken into consideration. On the other hand, the parameter $\alpha(t)$ is introduced into (15) to include the influence of diffraction loss.

The output power $P_{h \nu}$ of the device is given by

$$
P_{h \nu}=\frac{1}{2} \nu_{g} h \nu\left(1-\left|R_{0}\right|^{2}\right) \Gamma_{z} \pi W^{2} S \sum_{i} \int_{0}^{2 \pi} \int_{0}^{W}|\Psi|^{2} r d r d \theta
$$

where $h$ is the Planck's constant, $\nu$ is the operating frequency, and $R_{0}$ is the Bragg's reflectivity of the fundamental mode. The current spreading from a circular-disc contact along the active region can be expressed as [17]

$$
J(r, t)= \begin{cases}J_{o}(t) & r \leq W \\ J_{o}(t) \exp \left(-(r-W) / r_{o}\right), & r>W\end{cases}
$$

where $J_{o}$ denotes the current density at the edge within the contact area $(r<W)$, and $r_{o}$ is the effective-diffusion length of the injection carrier. In the photon rate equation, the effective optical gain $G$ is given by

$$
G=\frac{2}{W^{2}} \int_{0}^{\infty} g(N)|\Psi(r, t)|^{2} r d r .
$$

The effective material loss in the active layer, $\alpha_{m}$, and the spontaneous emission rate, $R_{\mathrm{sp}}$, are given by

$$
\begin{aligned}
\alpha_{m}(t) & =\frac{2}{W^{2}} \int_{0}^{\infty} \alpha_{m}^{\prime}(r)|\Psi(r, t)|^{2} r d r \\
R_{\mathrm{sp}}(t) & =\frac{2}{W^{2}} \beta B \int_{0}^{W} N^{2}(r, t)|\Psi(r, t)|^{2} r d r
\end{aligned}
$$

where $\beta$ is the spontaneous emission factor and $B$ is the bimolecular carrier recombination coefficient. $\alpha_{m}^{\prime}$ is the lateral distribution of material loss and is equal to $20 \mathrm{~cm}^{-1}\left(25 \mathrm{~cm}^{-1}\right)$ for the as-growth (diffused) QW active layer. Symbols not mentioned have similar meaning as given in Section II-A.

The transient behavior of $N$ and $S$ is calculated from the coupled differential equations (14) and (15) by using the 
fourth-order Runge-Kutta numerical integration method. The lateral variation of carrier concentration is obtained by using the finite difference method subjected to the corresponding continuity and boundary conditions [16] such that the influence of spatial hole burning can be computed. The lateral distribution of $\Psi$ is also solved simultaneously with the variation of carrier concentration such that: 1) self-focusing and 2) diffraction loss (i.e., arising from the time-dependent cavity loss, $\alpha(t))$ can be taken into consideration.

From the numerical model of VCSEL's developed in this section, the corresponding condition of SSP including spatial hole burning, self-focusing, and diffraction loss is given by

$$
\eta=\left(\bar{G}_{\mathrm{co}}-\bar{G}_{\mathrm{cl}}\right) \frac{\partial \Gamma}{\partial \bar{N}}+\left(\Gamma_{\mathrm{co}} \frac{\partial \bar{G}_{\mathrm{co}}}{\partial \bar{N}_{\mathrm{co}}}-\bar{\gamma} \Gamma_{\mathrm{cl}} \frac{\partial \bar{G}_{\mathrm{cl}}}{\partial \bar{N}_{\mathrm{cl}}}\right)-\frac{\partial \alpha}{\partial \bar{N}}<0
$$

where the subscript co stands for the core region and cl stands for the cladding region of the VCSEL's. $\bar{G}$ and $\partial \bar{G} / \partial \bar{N}$ are the equivalent optical gain and differential gain, respectively. $\Gamma$ is the optical confinement, $\bar{N}$ is the equivalent carrier concentration, and $\bar{\gamma}=\left|\bar{G}_{\mathrm{cl}} / \bar{G}_{\mathrm{co}}\right| . \partial \Gamma / \partial \bar{N}$ and $\partial \alpha / \partial \bar{N}$ are the equivalent differential confinement factor and cavity loss.

\section{Differential Confinement Factor and Differential Cavity Loss}

The optical confinement factors in the core and cladding region, $\Gamma_{c o}$ and $\Gamma_{c l}$, are defined as

$$
\Gamma_{\mathrm{co}}=\int_{0}^{W}|\Psi(r)|^{2} r d r / \int_{0}^{\infty}|\Psi(r)|^{2} r d r
$$

and

$$
\Gamma_{\mathrm{cl}}=1-\Gamma_{\mathrm{co}}
$$

Owing to the cavity-mirror interface structure and the modal nature of the lateral fields, the Bragg reflectivity can be computed by a self-consistent model-matching method [11] with the consideration of Bragg pairs, the cavity dimension, and the profile of the lateral fields. Fig. 1 shows the schematic of the Bragg reflector used in the calculation. It is assumed that the Bragg reflector has a circular symmetry with 30-pair GaAs-AlAs Bragg stack of length half wavelength each. The reflectivity of the fundamental mode (i.e. $\mathrm{LP}_{01}$ mode), $R_{0}$, is given by [11]

$$
\begin{aligned}
R_{0}= & \int_{0}^{\infty} \frac{\beta_{0}-H}{\beta_{0}+H} \Phi_{0} \Phi_{0}^{*} d k \\
& -\sum_{p \neq 0}^{\infty} R_{p} \int_{0}^{\infty}\left(\frac{\beta_{p}+H}{\beta_{0}+H}\right) \Phi_{p} \Phi_{0}^{*} d k
\end{aligned}
$$

where $H$ is the transfer function of the Bragg reflector in $k$ space, $\Phi_{p}(k)$ and $\beta_{p}$ are the first-order Hankel transform and propagation coefficient of the $p$ th mode. $R_{p}$ in (23) is given by

$$
\begin{aligned}
R_{p}= & \int_{0}^{\infty}\left\{\frac{\beta_{0}-H}{\beta_{p}+H}-R_{0} \frac{\beta_{0}+H}{\beta_{p}+H}\right\} \Phi_{0} \Phi_{p}^{*} d k \\
& -\sum_{q \neq 0, p}^{\infty} R_{q} \int_{0}^{\infty}\left(\frac{\beta_{q}+H}{\beta_{p}+H}\right) \Phi_{q} \Phi_{p}^{*} d k
\end{aligned}
$$

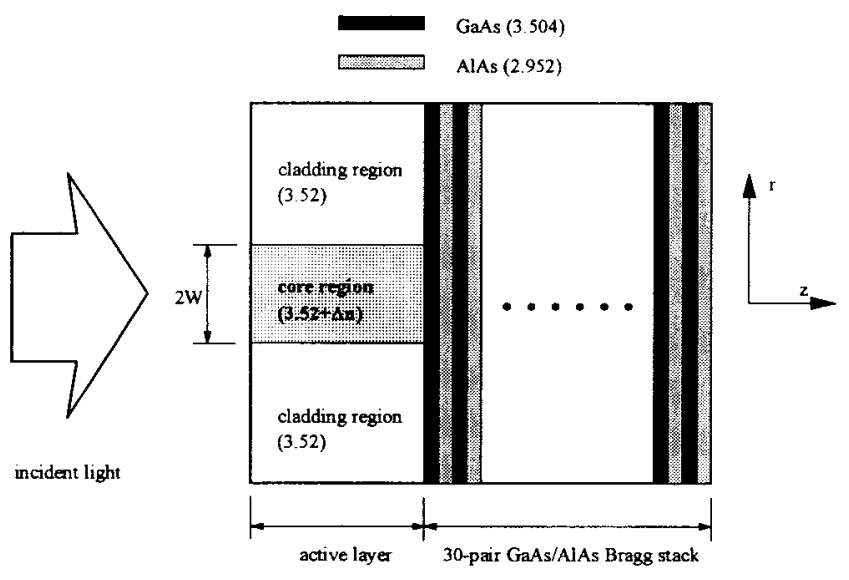

Fig. 1. Schematic of the Bragg reflector used to calculate the Bragg reflectivity under the influence of diffraction. $\Delta n$ is the difference of complex refractive index between the core and cladding regions.

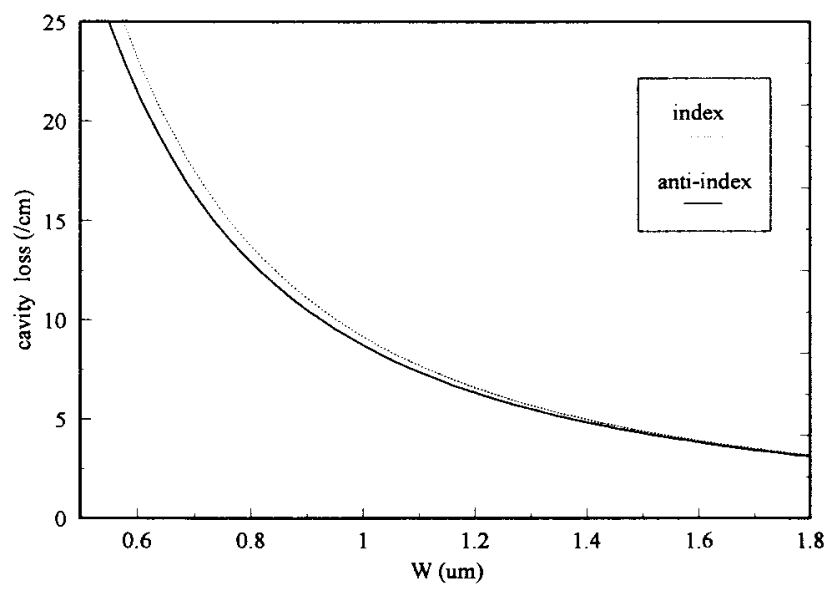

Fig. 2. Variation of cavity loss, $\alpha$, with core radius, $W$, for devices of index-guiding (dotted line) and anti-index-guiding (solid line) structures.

where $q$ is an integer. Therefore, the cavity loss, $\alpha$, for the fundamental mode in VCSEL's, is given by

$$
\alpha=\frac{1}{L_{\text {eff }}} \log \left(1 /\left|R_{0}\right|\right)
$$

where $L_{\text {eff }}$ is the effective cavity length.

Fig. 2 shows the variation of cavity loss with the core radius of VCSEL's. It is assumed that the VCSEL with a weak index-guiding structure has $\Delta n=0.0225$, and those with antiindex guiding structure has $\Delta n=-0.0225+j 0.012$, where $j=\sqrt{-1}$ and $\Delta n$ is the difference of complex refractive index between the core and cladding regions. The lateral distribution of the complex refractive index is assumed to be uniform along the core and cladding regions. As we expected, the cavity loss (i.e., arising from the diffraction effects) is negligible for $W>2 \mu \mathrm{m}$ but increases dramatically for $W<1 \mu \mathrm{m}$.

The differential confinement factor, $\partial \Gamma / \partial \bar{N}$, and the differential cavity loss, $\partial \alpha / \partial \bar{N}$, can be approximated as follows:

and

$$
\frac{\partial \Gamma}{\partial \bar{N}} \approx \frac{\Delta \Gamma}{\overline{\Delta N}} \approx \frac{2 \Delta \Gamma}{\overline{\Delta N}_{\mathrm{co}}-\overline{\Delta N}_{\mathrm{cl}}}
$$

$$
\frac{\partial \alpha}{\partial \bar{N}} \approx \frac{\Delta \alpha}{\overline{\partial N}} \approx \frac{2 \Delta \alpha}{\overline{\Delta N_{\mathrm{co}}-\overline{\Delta N}_{\mathrm{cl}}}}
$$




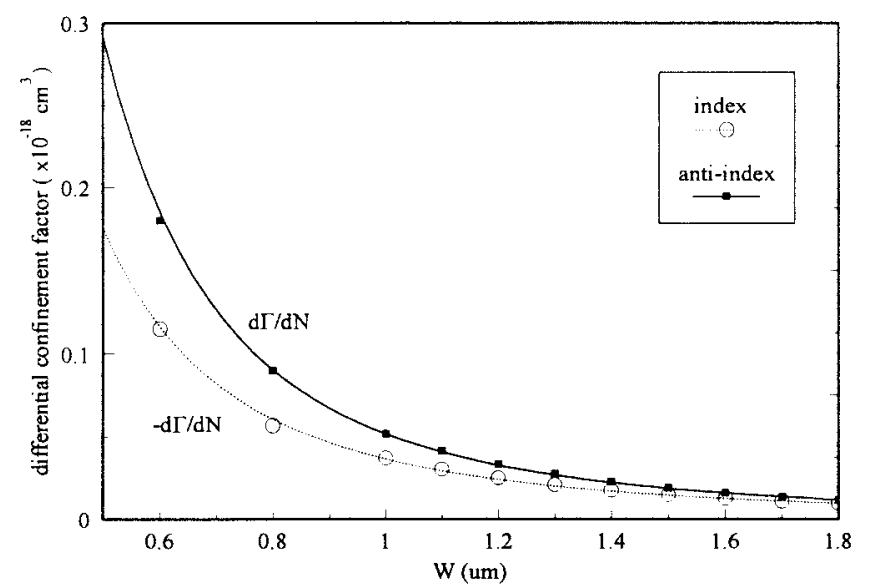

(a)

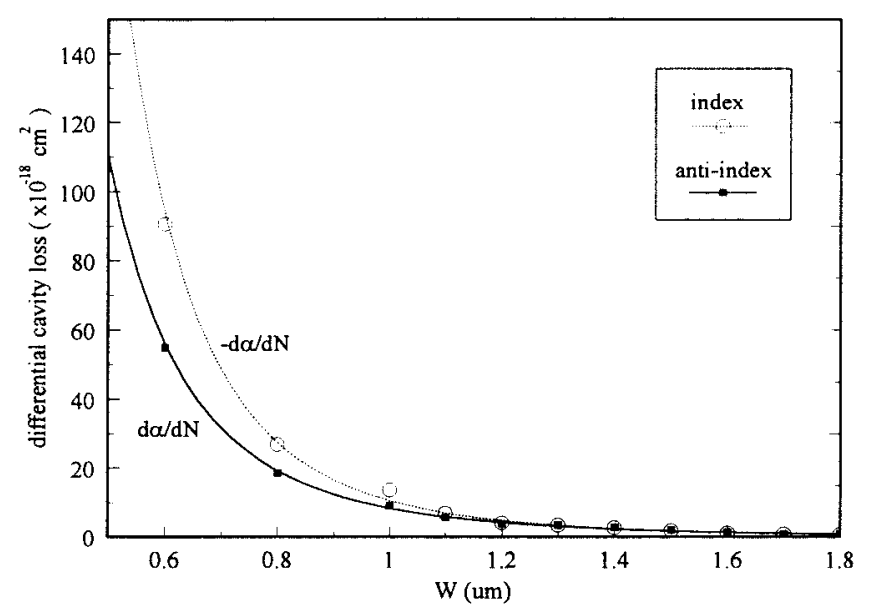

(b)

Fig. 3. Variation of (a) $\partial \Gamma / \partial \bar{N}$ and (b) $\partial \alpha / \partial \bar{N}$ with $W$, for devices of index-guiding (dotted line) and anti-index-guiding (solid line) structures.

where $\overline{\Delta N}_{\mathrm{co}}$ and $\overline{\Delta N}_{\mathrm{cl}}$ are the average change of carrier concentration inside the core and cladding regions. Fig. 3 shows the variation of $\partial \Gamma / \partial \bar{N}$ and $\partial \alpha / \partial \bar{N}$ of VCSEL's with weak index guiding structure (i.e., $\Delta n=0.0225$ ) and antiindex guiding structure (i.e. $\Delta n=-0.0225+j 0.012$ ) for different values of $W$. In the calculation, the variation of $\Delta n$ is related to the carrier concentration by (A4) of Appendix A.

In Fig. 3(a), the variation of $-\partial \Gamma / \partial \bar{N}$ for the index guiding device and $\partial \Gamma / \partial \bar{N}$ for the anti-index guiding device are plotted in Fig. 3(a) by varying $W$. As we can see, the device with index-guiding structure gives a negative value of $\partial \Gamma / \partial \bar{N}$ but that with an anti-guiding structure gives a positive value of $\partial \Gamma / \partial \bar{N}$. In addition, the absolute magnitude of $\partial \Gamma / \partial \bar{N}$ for both types of lateral guiding structure increases dramatically with the reduction of $W$. On the other hand, the variation of $-\partial \alpha / \partial \bar{N}$ for the index-guiding device and $\partial \alpha / \partial \bar{N}$ for the anti-index-guiding device are plotted in Fig. 3(b) by varying $W$. It is noted that the sign of $\partial \alpha / \partial \bar{N}$ is similar to that of $\partial \Gamma / \partial \bar{N}$ and the absolute magnitude of $\partial \alpha / \partial \bar{N}$ is increased with the reduction of $W$.

It is shown in Fig. 3 that the condition of SSP can be enhanced by $\partial \Gamma / \partial \bar{N}$ for the index-guiding structure or $\partial \alpha / \partial \bar{N}$ for the anti-index-guiding structure. However, it is not reliable to optimize the magnitude of $\partial \Gamma / \partial \bar{N}$ or $\partial \alpha / \partial \bar{N}$ to enhance SSP operation in VCSEL's because: 1) the magnitude of $\partial \Gamma / \partial \bar{N}$ and $\partial \alpha / \partial \bar{N}$ is also dependent on the lateral carrier distribution inside the active layer; 2) the influence of $\partial \Gamma / \partial \bar{N}$ is also affected by magnitude of optical gain difference [see (21)]; and 3) the magnitude of $\partial \Gamma / \partial \bar{N}$ and $\partial \alpha / \partial \bar{N}$ is only significant for small $W$ (i.e., $<1 \mu \mathrm{m}$ ). In fact, it is more practical to control the lateral overlap effects to enhance SSP in VCSEL's, provided that the lateral loss effects can be minimized. This is because the lateral loss effects are difficult to control and can deteriorate the SSP operation of VCSEL's. Therefore, a novel devices structure, with: 1) immunity to diffraction loss and self-focusing effect and 2) adjustable overlap volume between the saturable absorption region and the lasing mode, is required. These can be achieved by using a DFQW structure.

\section{VCSEL'S WITH DFQW STRUCTURE}

\section{A. Optical Gain Spectrum and Refractive Index Profile of Diffused Quantum Wells}

In this section, the optical properties of the DFQW material are studied. The models given in [18] and [19] are utilized to calculate the optical gain and the refractive index of the DFQW material. In the models, the extent of interdiffusion is characterized by a diffusion length of impurities $L_{d}$, where $L_{d}=0 \AA$ represents the as-grown QW's, and the diffusion strength is increased with the magnitude of $L_{d}$. It is assumed that the QW's active layer consists of six GaAs- $\mathrm{Al}_{0.3} \mathrm{Ga}_{0.7} \mathrm{As}$ QW's with well width of $100 \AA$ and the barrier thickness of $150 \AA$. Fig. 4(a) shows the optical gain spectrum of QW material (with the external injection carrier concentration of 3 $\times 10^{18} \mathrm{~cm}^{-3}$ ) by varying $L_{d}$. The corresponding background (under no external carrier injection) refractive index spectrum, $n_{B}(\lambda)$, of the QW's material is also shown in Fig. 4(b).

It is shown in Fig. 4(a) that at a particular $L_{d}$ and an operating wavelength $\lambda_{o}$, the optical gain, $g$, can be approximated by a simple relationship with the carrier concentration given as

$$
g(N)=a_{N}\left(\lambda_{o}\right) \log \left(N / N_{o}\left(\lambda_{o}\right)\right)
$$

where $a_{N}\left(\lambda_{o}\right)$ is the gain coefficient and $N_{o}\left(\lambda_{o}\right)$ is the carrier concentration at transparency. The change of refractive index, $\Delta n$, inside the QW's active layer can be evaluated from the change of gain spectral through the Kramers-Kronig dispersion relation [20]. We can also show that at a particular $L_{d}$ and $\lambda_{o}$, the relation between $\Delta n$ and $N$ can be expressed as

$$
\Delta n\left(\lambda_{\circ}\right)=d_{1}\left(\lambda_{\circ}\right) \log \left(N / N_{r}\left(\lambda_{o}\right)\right)
$$

where $d_{1}\left(\lambda_{o}\right)$ and $N_{r}\left(\lambda_{o}\right)$ are fitting parameters. If we assume the operation wavelength $\left(\lambda_{\circ}\right)$ of the devices to be equal to $0.85 \mu \mathrm{m}$ and $L_{d}$ of the active layer to be varied between 0-10 $\AA$, the magnitudes of $n_{B}, a_{N}, N_{o}, d_{1}$, and $N_{r}$ can be estimated from Fig. 4. 


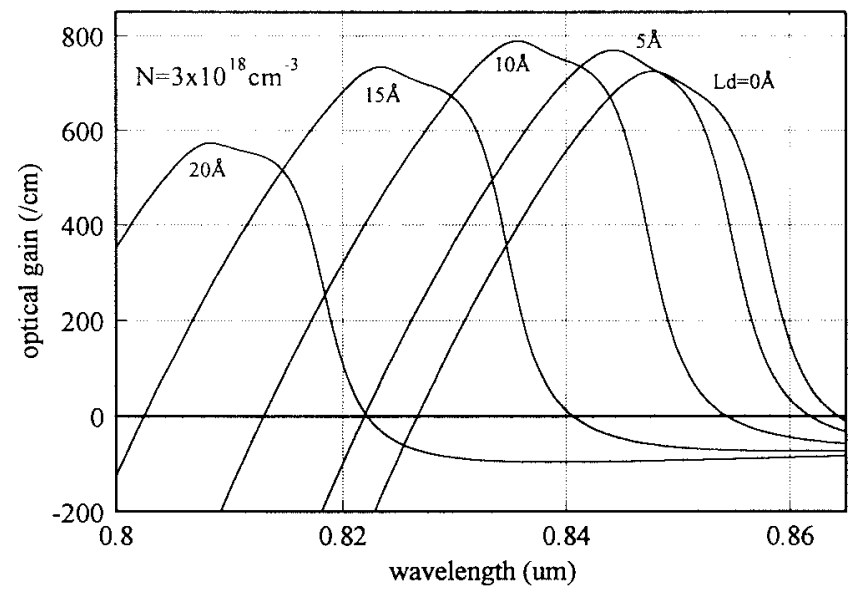

(a)

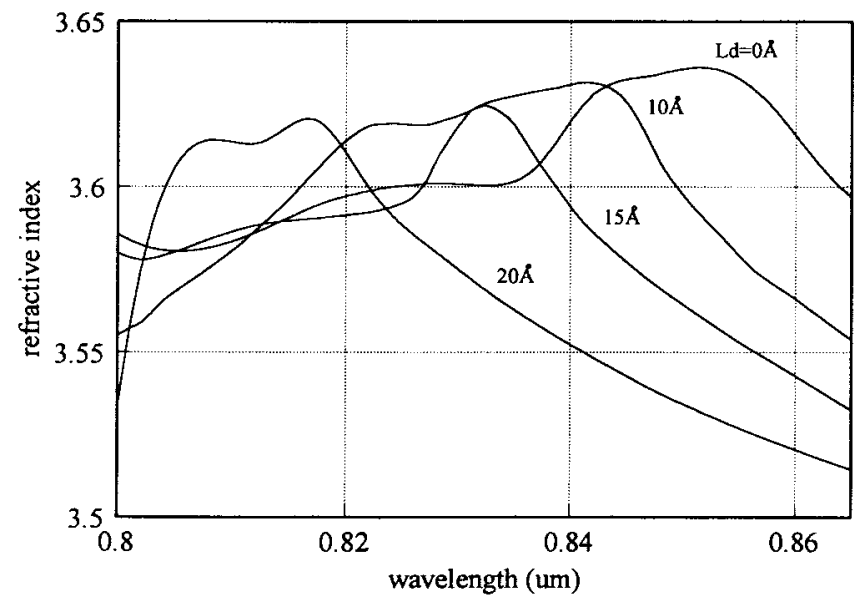

(b)

Fig. 4. Calculated (a) optical gain (with external injection carrier concentration of $3 \times 10^{18} \mathrm{~cm}^{-3}$ ) and (b) background refractive index of $\mathrm{Al}_{0.3} \mathrm{Ga}_{0.7}$ As-GaAs QW material with various levels of $L_{d}$.

\section{B. Proposed DFQW Structure for VCSEL's}

The lateral guidance of optical modes can be obtained in VCSEL's by applying interdiffusion into the QW's active layer, as shown in Fig. 5(a). A circular mask is utilized to shield the implanted ions on the as-grown QW's active layers. After implantation, annealing is applied to restore the impurities damage as well as to induce compositional disordering. The area covered by the mask forms the cladding region and the diffused area forms the core region of the VCSEL's. As a result, a small refractive index step between the core and cladding region is established at the QW's active layer such that an anti-index-guided structure is obtained. The spacer layer, p-type Bragg reflector, and circular metal contact will then be grown on the DFQW's active layer to form a complete structure of VCSEL's. Fig. 5(b) shows the proposed VCSEL with the DFQW structure.

The purpose of using the DFQW structure is: 1) to maximize the lateral overlap effects and 2) to minimize the lateral loss effects. This can be explained as follows.

- The background refractive index reduces with the increase of $L_{d}$ at $\lambda_{o}$ equals $0.85 \mu \mathrm{m}$. Therefore, the laser with diffusion applied into the core region of the QW active

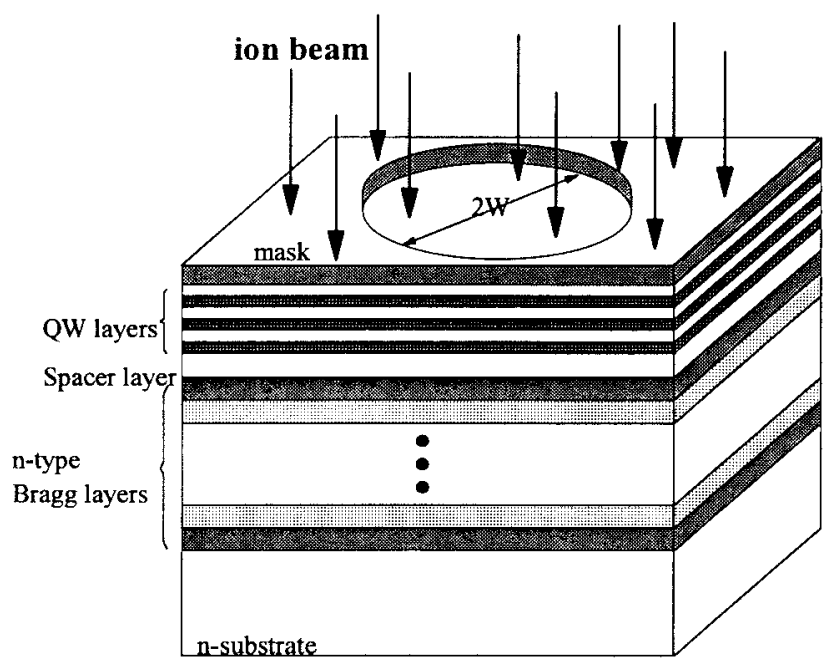

(a)

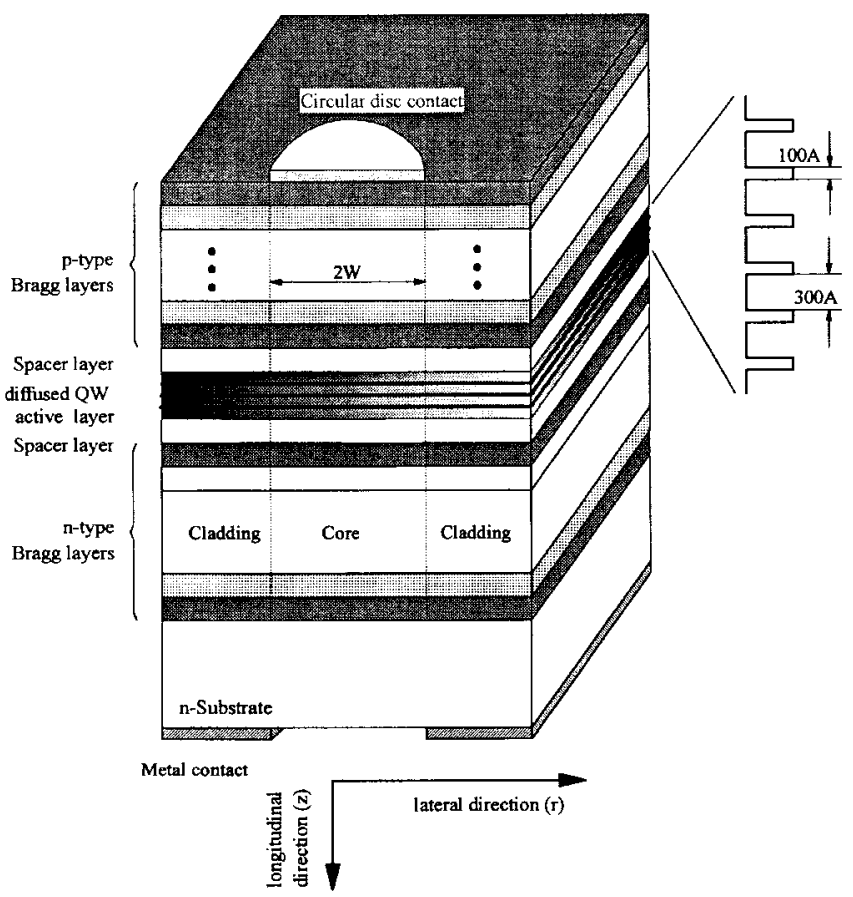

(b)

Fig. 5. (a) Ion implantation on a QW's layers of a VCSEL with a hollow circular mask of radius $W$. (b) Schematic structure of a VCSEL with DFQW structure.

layer forms an anti-guiding structure for the lateral mode such that it can be further penetrated into the cladding region to enhance optical absorption.

- The absorption peak wavelength of the as-grown QW's is a little longer than that of the DFQW's such that the saturable absorption is increased.

- The differential gain $\left(=a_{N} / N\right)$ of the core region is reduced by interdiffusion such that the condition of SSP is enhanced.

- SSP can be maintained in the anti-guiding structure with wide core radius such that the influence of lateral loss effects is reduced.

In order to maximize the overlap volume between the saturable absorption region and the lasing mode, a large value 
TABLE I

Parameters for the QW Material

\begin{tabular}{cccccc}
\hline$L_{d}(\AA)$ & $n_{\beta}\left(\lambda_{o}\right)$ & $\begin{array}{c}a_{N}\left(\lambda_{o}\right) \\
\left(\mathrm{cm}^{-1}\right)\end{array}$ & $\begin{array}{c}N_{o} \times 10^{18} \\
\left(\mathrm{~cm}^{-3}\right)\end{array}$ & $d_{1}\left(\lambda_{0}\right)$ & $\begin{array}{c}N_{r}\left(\lambda_{o}\right) \times 10^{18} \\
\left(\mathrm{~cm}^{-3}\right)\end{array}$ \\
\hline 0 & 3.6270 & 1780.0121 & 2.1996 & -0.02824 & 2.0673 \\
5 & 3.6074 & 1507.6078 & 2.0515 & -0.02777 & 2.0164 \\
10 & 3.5820 & 468.6990 & 2.4092 & -0.02521 & 1.9809 \\
\hline
\end{tabular}

TABLE II

PARAMETERS FOR THE VCSEL'S

\begin{tabular}{|c|c|c|}
\hline Parameter & Symbol & Value \\
\hline operating wavelength & $\lambda_{o}$ & $0.85 \mu \mathrm{m}$ \\
\hline group velocity of light & $v_{g}$ & $0.83 \times 10^{10} \mathrm{~cm} / \mathrm{s}$ \\
\hline longitudinal optical confinement factor & $\Gamma_{z}$ & 0.16 \\
\hline $\begin{array}{c}\text { bimolecular carrier recombination } \\
\text { coefficient }\end{array}$ & $B$ & $1 \times 10^{-10} \mathrm{~cm}^{3} \cdot \mathrm{s}^{-1}$ \\
\hline spontaneous emission factor & $\beta$ & $1 \times 10^{-4}$ \\
\hline carrier lifetime in core region & $\tau_{1}$ & $4 \times 10^{-9} \mathrm{~s}$ \\
\hline carrier lifetime in cladding region & $\tau_{2}$ & $5 \times 10^{-9} \mathrm{~s}$ \\
\hline effective cavity length & $L_{\text {eff }}$ & $1.4 \mu \mathrm{m}$ \\
\hline diffusion coefficient & $D$ & $5 \mathrm{~s}^{-1} \cdot \mathrm{cm}^{2}$ \\
\hline $\begin{array}{l}\text { effective diffusion length of the injection } \\
\text { carrier }\end{array}$ & $r_{0}$ & $0.01 \mu \mathrm{m}$ \\
\hline thickness of active layer & $d$ & $0.15 \mu \mathrm{m}$ \\
\hline
\end{tabular}

of $L_{d}$ is preferred. However, in a practical situation, $L_{d}$ should not be larger than $10 \AA$ so that the optical gain inside the core region can be preserved at a reasonable injection carrier concentration.

\section{Simulation Results}

The parameters for optical gain and refractive index of the DFQW's material used in the calculation can be found in Table I. Other devices parameters used in the model are shown in Table II.

\section{A. Influence of Lateral Loss on the Generation Mechanism of SSP}

Fig. 6 plots the variation of instability constant $\eta$ as a function of $W$. It is assumed that the diffusion is applied to the core region of the active layer with $L_{d}$ varying between 0 and $10 \AA$. The injection current density of the devices is set to about 1.1 times its threshold value. In addition, the curves at $L_{d}=10 \AA$ without the inclusion of $\partial \Gamma / \partial \bar{N}$ and $\partial \alpha / \partial \bar{N}$ are also shown in the figure for comparison. The following is shown.

1) The range of $W$ (for $\eta<0$ ) increases with $L_{d}$ due to the enhancement of absorption loss inside the cladding region.

2) The lateral loss effects (i.e., $\partial \Gamma / \partial \bar{N}$ and $\partial \alpha / \partial \bar{N}$ ) have significant influence on the instability constant $\eta$ only for small $W$.

3) For devices with large $W$, the instability constant $\eta$ is mostly affected by the saturable absorption inside the core region.

The results indicate that the influence of lateral loss effects in VCSEL's can be minimized by using our proposed DFQW structure.

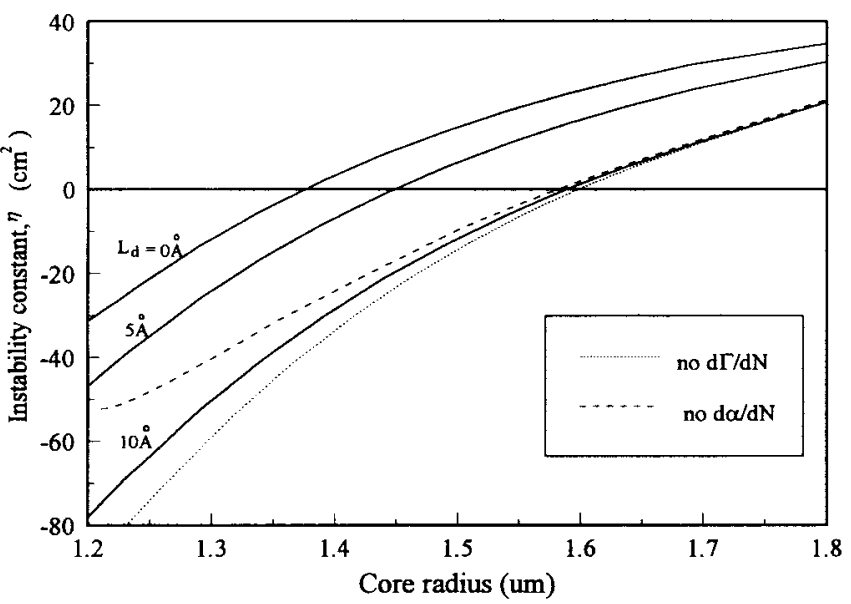

Fig. 6. Variation of instability constant $\eta$ with $W$ and $L_{d}$. The lasers are assumed biased at 1.1 times its threshold value.

\section{B. Pulse Repetition Frequency and Peak Pulse Power}

The current dependency of pulse repetition frequency and peak pulse power are shown in Fig. 7. It is assumed that the core radius of the devices is equal to $1.3 \mu \mathrm{m}$ with $L_{d}$ varying between 0 and $10 \AA$. It is observed that the pulse repetition frequency (for both SSP and relaxation oscillation) as well as the peak output power increase with $L_{d}$. As we can see, the condition to get SSP operation with high oscillation frequency and peak power are: 1) low optical confinement and 2) small differential gain inside the core region of the active layer. This is because the refractive index and differential gain coefficient are reduced with increase of $L_{d}$.

\section{DISCUSSION AND CONCLUSION}

The main advantage of using DFQW structures in VCSEL's to generate SSP is the precise control of overlap volume between the gain-absorber regions and lasing mode. However, the controllability of using diffusion is limited by the precision of the dimensions of $L_{d}$ and $W$ that can be obtained. It must be noted that the magnitude of $L_{d}$ is affected by the implantation energy and annealing time of the interdiffusion process. With careful control of annealing temperature and time, $L_{d}$ down to $5 \AA$ with error of $\pm 1 \AA$ can be achieved without any difficulty. Furthermore, the combined technologies of electron beam lithography and implanted enhanced intermixing [21] are utilized to realize a structure which is far more precise than our requirement to define core radius. Therefore, diffusion is a much better method to obtain SSP with good reproducibility. One drawback in making VCSEL's using diffusion is that the absorption and scattering losses inside the active layer may increase due to the damage of lattice by the implanted ions. However, if low implantation energy is used, serious degradation of the device's performance can be avoided. This is because low-energy implanted ions minimize the lattice damage due to the collision and the defect can be easily recovered by thermal annealing. In fact, implantation-enhanced intermixing has been utilized to fabricate QW semiconductor lasers which exhibit no serious deterioration of the device's performance [22]. Therefore, with careful control of implan- 


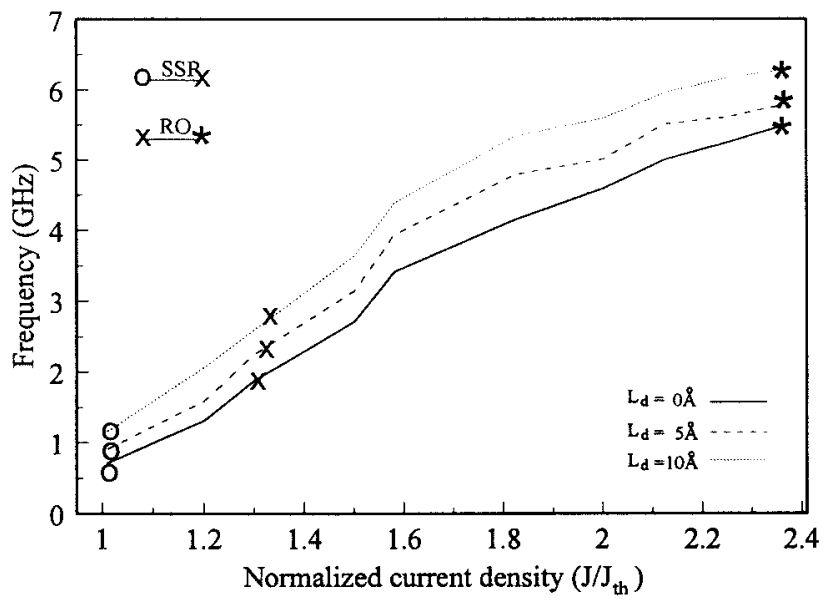

(a)

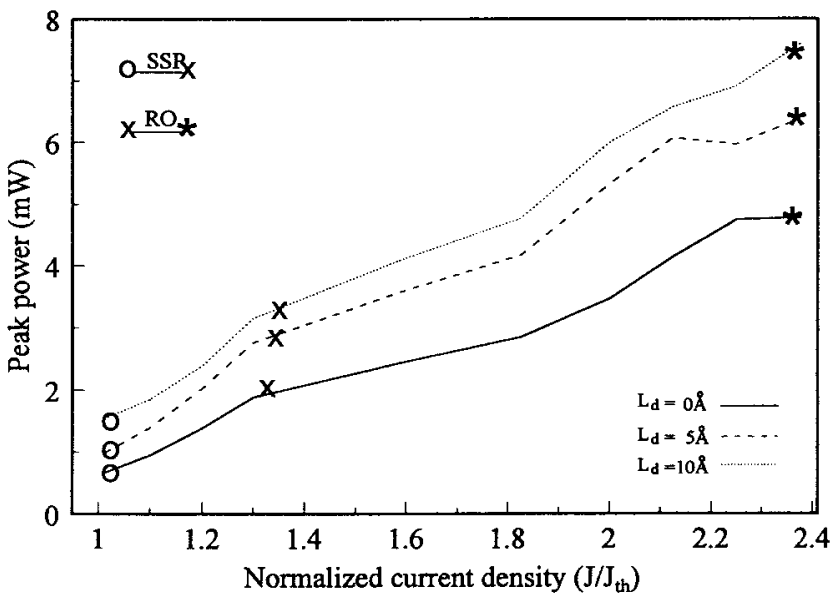

(b)

Fig. 7. Current dependence of (a) pulse repetition frequency and (b) output power for VCSEL's with $W=1.3 \mu \mathrm{m}$ and $L_{d}=0,5$, and $10 \AA$.

tation energy and annealing time, the standard performance of VCSEL's can be maintained.

In conclusion, the generation mechanism of SSP in VCSEL's is clarified. It is shown that lateral loss effects have significant influence on the generation mechanism of SSP, especially for VCSEL's with small size (i.e., $W<1 \mu \mathrm{m}$ ). Furthermore, the DFQW's structure for VCSEL's is proposed to enhance SSP. It is shown that the proposed DFQW's structure enhances the lateral overlap effects but minimizes the influence of lateral loss effects. This is because the DFQW's structure maximizes the overlap volume between the absorption region and lasing mode as well as the core radius of the VCSEL. In addition, peak power and high repetition frequency are also improved. Therefore, the possibility of using DFQW's structure to obtain SSP in VCSEL's is verified and it is believed that the DFQW structure can also be applied to facet-emitted lasers for the enhancement of SSP.

\section{APPENDIX A}

In the model, the weakly guided lateral fields are expressed in terms of linearly polarized $\left(\mathrm{LP}_{l p}\right)$ modes [23] where the indexes $l$ and $p$ denote the azimuthal and radial order of modes, respectively. The lateral field, $\Psi$, is calculated from the complex scalar Helmholtz [23], given as

$$
\frac{\partial^{2} \Psi}{\partial r^{2}}+\frac{1}{r} \frac{\partial \Psi}{\partial r}+\frac{1}{r} \frac{\partial^{2} \Psi}{\partial \theta^{2}}+\frac{\partial^{2} \Psi}{\partial z^{2}}+\varepsilon k_{0}^{2} \Psi=0
$$

where $\theta$ is the azimuthal angle and $z$ and $r$ are the longitudinal and lateral coordinates, respectively. In order to consider the nonuniform distribution of permittivity, the active layer is subdivided into small equal segments along the lateral direction with uniform permittivity inside each segment. Therefore, the solution to (A1) can be expressed by the Bessel functions $J_{l}, Y_{l}$ and modified Hankel functions $K_{l}$ of order $l$ as in (A2), shown at the bottom of the page, where $j=\sqrt{-1}, r_{m}=m \Delta r$ and $\Delta r=W / M$ such that $r_{M}=W$ and $r_{2 M}=2 W$.

The parameters $\kappa$ and $\gamma$ are defined by

and

$$
\begin{aligned}
& \kappa^{2}\left(r_{m}\right)=\varepsilon\left(r_{m}\right) k_{0}^{2}-\beta^{2} \\
& \gamma^{2}\left(r_{m}\right)=\beta^{2}-\varepsilon\left(r_{m}\right) k_{0}^{2} .
\end{aligned}
$$

The effective permittivity in (A3) is given by

$$
\begin{aligned}
\varepsilon\left(r_{m}\right)= & n_{B}^{2}\left(r_{m}\right)-2 n_{B}\left(r_{m}\right) \frac{\partial n}{\partial N} N\left(r_{m}\right) \\
& +j \frac{n_{B}\left(r_{m}\right)}{k_{o}}\left[a_{N}\left\{N\left(r_{m}\right)-N_{o}\right\}-\alpha_{m}\left(r_{m}\right)\right\} \text { (A4) }
\end{aligned}
$$

where $n_{B}$ is the built-in refractive index, $k_{o}\left(=2 \pi / \lambda_{0}\right)$ is the free-space wavenumber and $\partial n / \partial N$ is the change in refractive index by carrier concentration. Matching of $\Psi$ and its derivative at the zone boundaries (i.e., $r_{1}, r_{2}, \cdots, r_{2 M}$ ) gives a set of linear homogeneous equations for the complex coefficient $A_{m}$ and $B_{m}$ (for $m=1,2, \cdots, 2 M+1$ ). The condition for nontrivial solutions determines the complex propagation coefficient $\beta$ and the field profile of $\Psi$.

$$
\Psi=\exp \left(j_{l} \theta\right) \exp (j \beta z)\left\{\begin{array}{cc}
A_{1} J_{l}\left(\kappa_{1} r\right) & 0 \leq r<r_{1} \\
A_{2} J_{l}\left(\kappa_{2} r\right)+B_{2} Y_{l}\left(\kappa_{2} r\right) & r_{1} \leq r<r_{2} \\
\vdots & \\
A_{M} J_{l}\left(\kappa_{M} r\right)+B_{M} Y_{l}\left(\kappa_{M} r\right) & r_{M-1} \leq r<r_{M} \\
A_{M+1} K_{l}\left(\gamma_{M+1} r\right)+B_{M+1} I_{l}\left(\gamma_{M+1} r\right) & r_{M} \leq r<r_{M+1} \\
\vdots & \\
A_{2 M} K_{l}\left(\gamma_{2 M} r\right)+B_{2 M} I_{l}\left(\gamma_{2 M} r\right) & r_{2 M-1} \leq r<r_{2 M} \\
A_{2 M+1} K_{l}\left(\gamma_{2 M+1} r\right) & r>2 w
\end{array}\right.
$$




\section{ACKNOWLEDGMENT}

The author would like to thank K. Chui of HKUEEE for his computational work and the reviewer for his/her constructive comments.

\section{REFERENCES}

[1] K. P. Ho, J. D. Walker, and J. M. Kahn, "External optical feedback effects on intensity noise of vertical cavity surface emitting lasers," IEEE Photon. Technol. Lett., vol. 5, pp. 892-895, 1993.

[2] S. Matsui, H. Takiguchi, H. Hayashi, S. Yamamoto, S. Yano, and T. Hijikata, "Suppression of feedback-induced noise in short V-channeled substrate inner stripe lasers with self-oscillation," Appl. Phys. Lett., vol. 43, pp. 219-221, 1983.

[3] M. Yamada and T. Higashi, "Mechanism of the noise reduction method by superposition of high frequency current for semiconductor injection lasers," IEEE J. Quantum Electron., vol. 27, pp. 380-388, 1991.

[4] C. Z. Guo and K. G. Wang, "Intrinsic pulsation in stripe geometry DH semiconductor lasers," IEEE J. Quantum Electron., vol. QE-18, pp. $1728-1737,1982$

[5] B. Weigl, M. Grabherr, R. Michalzik, G. Reiner, and K. J. Ebeling, "High power single mode selectively oxidized vertical cavity surface emitting lasers," IEEE Photon. Technol. Lett., vol. 8, pp. 971-973, 1996.

[6] M. Orenstein, A. C. Von Lehmen, C. Chang-Hasnain, N. G. Stoffel, J. P. Harbison, L. T. Florez, E. Clausen, and J. E. Jewell, "Vertical cavity surface emitting InGaAs/GaAs lasers with planar lateral definition," Appl. Phys. Lett., vol. 56, no. 24, pp. 2384-2386, 1990.

[7] U. Fiedler, B. Moller, G. Reiner, D. Wiedenmann, and K. L. Ebeling, "Proton implanted VCSEL's for $3 \mathrm{~Gb} / \mathrm{s}$ data links," IEEE Photon. Technol. Lett., vol. 7, pp. 1116-1118, 1995.

[8] D. Vakhshoori, J. D. Wynn, G. J. Zydzik, R. E. Leibenguth, M. T. Asom, K. Kojima, and R. A. Morgan, "Top surface emitting lasers with $1.9 \mathrm{~V}$ threshold voltage and the effect of spatial hole burning on their transverse mode operation and efficiency," Appl. Phys. Lett., vol. 62, no. 13 , pp. 1448-1450, 1993.

[9] T. Wolf, C. L. Shieh, R. Engelmann, K. Alavi, and J. Mantz, "Lateral refractive index step in GaAs/AlGaAs multiple quantum well waveguides fabricated by impurity-induced disordering," Appl. Phys. Lett., vol. 55, pp. 1412-1414, 1989.

[10] S. F. Yu and E. H. Li, "Influence of lateral field on the relaxation oscillation frequency of semiconductor lasers," IEEE J. Quantum Electron. vol. 32, pp. 1-3, 1996.

[11] R. R. Burton, M. S. Stern, P. C. Kendall, and P. N. Robson, "Modeling of diffraction in pillar vertical cavity surface emitting lasers with embedded Bragg layers," Opt. Ouantum Electron., vol. 28, pp. 1677-1684, 1996.

[12] M. Yamada, "A theoretical analysis of self-sustained pulsation phenomena in narrow stripe semiconductor laser," IEEE J. Quantum Electron., vol. 29, pp. 1330-1336, 1993.

[13] M. Yuri, J. S. Harris, Jr., T. Takayama, O. Imafuji, H. Naito, M. Kume, K. Itoh, and T. Baba, "Two-dimensional analysis of self-sustained pulsation for narrow-stripe AlGaAs lasers," IEEE J. Select. Topics Quantum Electron., vol. 1, pp. 473-479, 1995.

[14] M. J. Adams, An Introduction to Optical Waveguide. New York: Wiley, 1981, ch. 2.
[15] H. Kawaguchi, "Absorptive and dispersive bistability in semiconductor injection lasers," Opt. Ouantum Electron., vol. 19, pp. S1-S36, 1987.

[16] S. F. Yu, "Dynamic behavior of vertical cavity surface emitting lasers," IEEE J. Quantum Electron., vol. 32, pp. 1168-1179, 1996.

[17] N. K. Dutta, "Analysis of current spreading, carrier diffusion and transverse mode guiding in surface emitting lasers," J. Appl. Phys., vol. 68, no. 5, pp. 1961-1963, 1990.

[18] E. H. Li and K. S. Chan, "Laser gain and current density in a disordered AlGaAs/GaAs quantum well," Electron. Lett., vol. 29, pp. 1233-1234, 1993.

[19] E. H. Li, B. L. Weiss, K. S. Chan, and J. Micallef, "The polarization dependent refractive index of an interdiffusion induced AlGaAs/GaAs Quantum well," Appl. Phys. Lett., vol. 62, pp. 550-552, 1992.

[20] C. H. Herny, R. A. Logan, and K. A. Bertness, "Spectral dependence of the change in refractive index due to carrier injection in GaAs lasers," J. Appl. Phys., vol. 52, pp. 4457-4461, 1981.

[21] C. Kaden, H. Grabeldinger, H. P. Gauggel, V. Hofsab, A. Hase, A. Menschig, H. Schweizer, R. Zengerle, and H. J. Bruckner, Microelectron. Eng., vol. 23, p. 469, 1994.

[22] V. Hofsäß, J. Kuhn, C. Kaden, V. Härle, H. Bolay, F. Scholz, H Schweizer, H. Hillmer, R. Lösch, and W. Schlapp, "Optical integration of laterally modified multiple quantum well structures by implantation enhanced intermixing to realize gain coupled DFB lasers," Nucl. Instrum. Method Phys. Res. B, vol. 106, pp. 471-476, 1995.

[23] A. W. Snyder and J. D. Love, Optical Waveguide Theory. London, U.K.: Chapman and Hall, 1983.

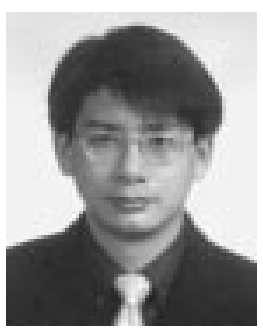

S. F. Yu received the B.Eng. degree (with Departmental Prize) in electronic engineering from London University, University College, England, in 1990 and the Ph.D. degree in sptoelectronics from Cambridge University, Robinson College, England, in 1993.

He joined the Department of Electronic Engineering, Sha Tin Technical Institute, Hong Kong, as a part-time lecturer in 1993. In 1994, he joined the Department of Electrical and Electronic Engineering, the University of Hong Kong, where he was a Lecturer. Since 1996, he has been an Assistant Professor in the same department of the University of Hong Kong. His main research topics include wavelength selectivity of grating coupled waveguides, transient properties of semiconductor laser diodes, and design of optoelectronics integrated circuit. $\mathrm{He}$ currently conducts the development of high-performance semiconductor lasers using diffused quantum-well material for the application in high-speed communication systems. He also involves the investigation of optical soliton fiber communication system. He has published over 60 technical papers and one book chapter.

Dr. Yu is a fellow and Honorary Scholar of Cambridge Commonwealth Trust Society. He held a Croucher Foundation scholarship and an overseas research student award while studying for the Doctoral program. His biography is published in the fourth edition of Who's Who in Science and Engineering (Marquis, USA), the 25th edition of Dictionary of International Biography and the 17th edition of International Directory of Distinguished Leadership. $\mathrm{He}$ is a member of the Optical Society of America. 